\title{
Perioperative complications and adverse sequelae of radical nephroureterectomy
}

\author{
Alison Levy, David Canes \\ Department of Urology, Lahey Hospital and Medical Center, Burlington, MA, USA \\ Contributions: (I) Conception and design: A Levy, D Canes; (II) Administrative support: None; (III) Provision of study material or patients: A Levy; \\ (IV) Collection and assembly of data: None; (V) Data analysis and interpretation: A Levy; (VI) Manuscript writing: All authors; (VII) Final approval \\ of manuscript: All authors. \\ Correspondence to: Alison Levy. Department of Urology, Lahey Hospital and Medical Center, Burlington, MA, USA. Email: ali.levy@gmail.com.
}

\begin{abstract}
Radical nephroureterectomy (RNU) has long been considered the standard of care for treatment of upper tract urothelial carcinoma (UTUC). Despite providing oncologic control, RNU is associated with measurable morbidity and mortality. High quality data is lacking as a result of low disease incidence and very few randomized studies. In this article we will review preoperative nomograms that assist with patient counseling, summarize current knowledge about perioperative complications, and discuss adverse sequelae that may result after surgery.
\end{abstract}

Keywords: Nephroureterectomy; morbidity; complications

Submitted Nov 13, 2019. Accepted for publication Dec 13, 2019.

doi: 10.21037/tau.2019.12.25

View this article at: http://dx.doi.org/10.21037/tau.2019.12.25

\section{Introduction}

Upper tract urothelial carcinoma (UTUC) is a rare but aggressive urologic malignancy with an incidence of less than 2 per 100,000. Detection rates have increased with improvements in imaging (1). Since the malignancy has a propensity to spread to nearby urothelium and to the bladder, maximal disease control is achieved by performing radical nephroureterectomy (RNU) with ipsilateral removal of a bladder cuff. RNU is recommended for non-metastatic high grade, bulky or invasive urothelial tumors and is a treatment option for low grade UTUC $(2,3)$.

Low disease incidence and heterogeneity of tumors at presentation has resulted in significant research limitations. Few randomized trials exist to guide management. Treatment has largely been extrapolated from understanding of lower tract urothelial lesions; however, upper and lower tract UC are distinct diseases. From a surgical standpoint, RNU is a more complex and morbid procedure compared to radical nephrectomy or segmental ureterectomy (SU). Understanding preoperative considerations, perioperative complications and adverse sequelae of RNU is essential for patient counseling.

\section{Preoperative considerations/nomograms}

RNU is associated with measurable morbidity and nonnegligible risk of mortality. Risk stratifying patients can be challenging but is essential to ensure they receive appropriate therapy. NCCN guidelines advise stratifying patients by grade and stage as well as tumor location (renal pelvis, proximal, mid, distal ureter) to determine optimal surgical management (3). Nephron sparing surgery is advised in patients with bilateral disease, or current or strong predisposition to renal dysfunction. EAU guidelines recommend offering kidney sparing surgery to patients with low risk tumors, high risk distal tumors, and patients with solitary kidney or impaired renal function if it will not compromise survival (4). Both sets of guidelines leave the patient and surgeon with several options to weigh after a new UTUC diagnosis.

Several tools are available to guide patients regarding perioperative expectations with respect to complications after RNU. Calculators related to oncologic outcomes are beyond the scope of this review.

The National Surgical Quality Improvement Program (NSQIP) surgical risk calculator is a robust tool maintained 
by the American College of Surgery to predict risk of 30-day overall and specific complications. The tool was built using data from over 4.3 million patients from 780 participating hospitals covering a wide geographical area and variety of practices. Between 2005-2013 the database included 912 patients who underwent open or minimally invasive RNU (5). Using this calculator, the overall risk of complications was $12.2-18.6 \%$ with risk of serious complications ranging from $11.3-18.2 \%$.

The most frequently identified complications in NSQIP were urinary tract infection, surgical site infection, sepsis and pneumonia. A modest 30-day readmission rate of $7.3-10.3 \%$ is noted. Rates of pulmonary embolism, pneumonia, and transfusion were found to be higher for patients undergoing open RNU compared to minimally invasive RNU (5). A more recent analysis including all patients undergoing RNU identified 1,443 patients and reported $1.7 \%$ 30-day mortality and $5.3 \%$ rate of ClavienDindo grade IV complications (6). The most common complications identified in that analysis were bleeding requiring blood transfusion, renal failure, surgical site infection and sepsis. This tool provides risk estimates specific patient characteristics, thereby offering a tailored approach when trying to counsel patients based on their overall and specific risk of complications.

Using the same NSQIP data, Lascano et al. created a modified frailty index $(\mathrm{mFI})$ based on the Canadian Study of Health and Aging Frailty Index to attempt to better predict adverse outcomes (6). The 15 variables incorporated can easily be assessed at a preoperative visit and were significantly associated with increased risk of 30-day mortality, Clavien-Dindo IV complications, surgical site infection, ventilator dependence, reintubation, myocardial infarction, acute renal failure, cardiac arrest, deep vein thrombosis, bleeding requiring blood transfusion and reoperation. However, the $\mathrm{mFI}$ was not superior to ASA classification in predicting mortality or severe complications but when combined with ASA was a superior predictor compared to ASA alone.

Recently, Raman et al. created a nomogram to predict perioperative complications in patients undergoing RNU for UTUC (7). The authors used data from 731 patients from 8 academic medical centers between 2002-2014. Charts were reviewed retrospectively for the purpose of the study, thereby decreasing the risk of coding errors inherent to larger databases. This study identified a significantly higher overall complication rate of $38.2 \%$ of which $22 \%$ were Clavien grade III or higher. Specific complications were not described, however a 30 -day mortality rate of $1 \%$ was noted, with most falling in hematologic, gastrointestinal or infectious categories. On multivariate analysis variables conferring higher risk of complication were age, race, ECOG performance status, Charlson Comorbidity Index score and CKD stage. Using these factors, the authors created a straightforward predictive tool that could be useful for preoperative counseling (Figure 1). This study is limited in its application to non-academic medical centers, however it is valuable as a tool developed uniquely for patients undergoing RNU.

\section{Preoperative interventions}

Limited urology-specific data is available regarding preoperative optimization. Knowing that patients with UTUC often have significant comorbidities, it would be useful to know which risk factors can be altered to improve post-operative outcomes and reduce complications (8). Well established interventions throughout the literature include medication optimization and smoking cessation. Nearly half of patients undergoing RNU present with anemia, owing to the presence of malignancy, poor nutritional status, and higher rates of preoperative renal disease, but inadequate knowledge regarding the potential impact of intervention exists (8). Blood transfusion carries significant drawbacks and is associated with postoperative mortality. The PREVENTT study, which is in its final stages of recruitment, will investigate the potential benefit of IV iron supplementation for patients undergoing major open abdominal surgery and may help to determine the value of correcting preoperative anemia in this population (9).

Surgery is a significant stressor and evidence has shown that patients with diminished tissue oxygen delivery and preoperative nutritional status fare worse. Recent research has sought to determine if preoperative rehabilitation, or "prehab" as well as optimizing nutrition status prepares patients for the insult of surgery and improves their recovery. To our knowledge, there has been no research investigating prehab among patients undergoing nephroureterectomy and studies from cystectomy literature have not shown measurable benefits from prehab programs (8).

Poor nutritional status has been shown to be a poor prognostic indicator in UTUC. In a single-center retrospective study by Huang et al the authors looked at 520 consecutive patients over the course of 10 years who underwent RNU for UTUC (10). Using preoperative albumin and prognostic nutritional index (PNI) which is calculated using albumin and blood counts, the authors found a significant association between PNI and lower 


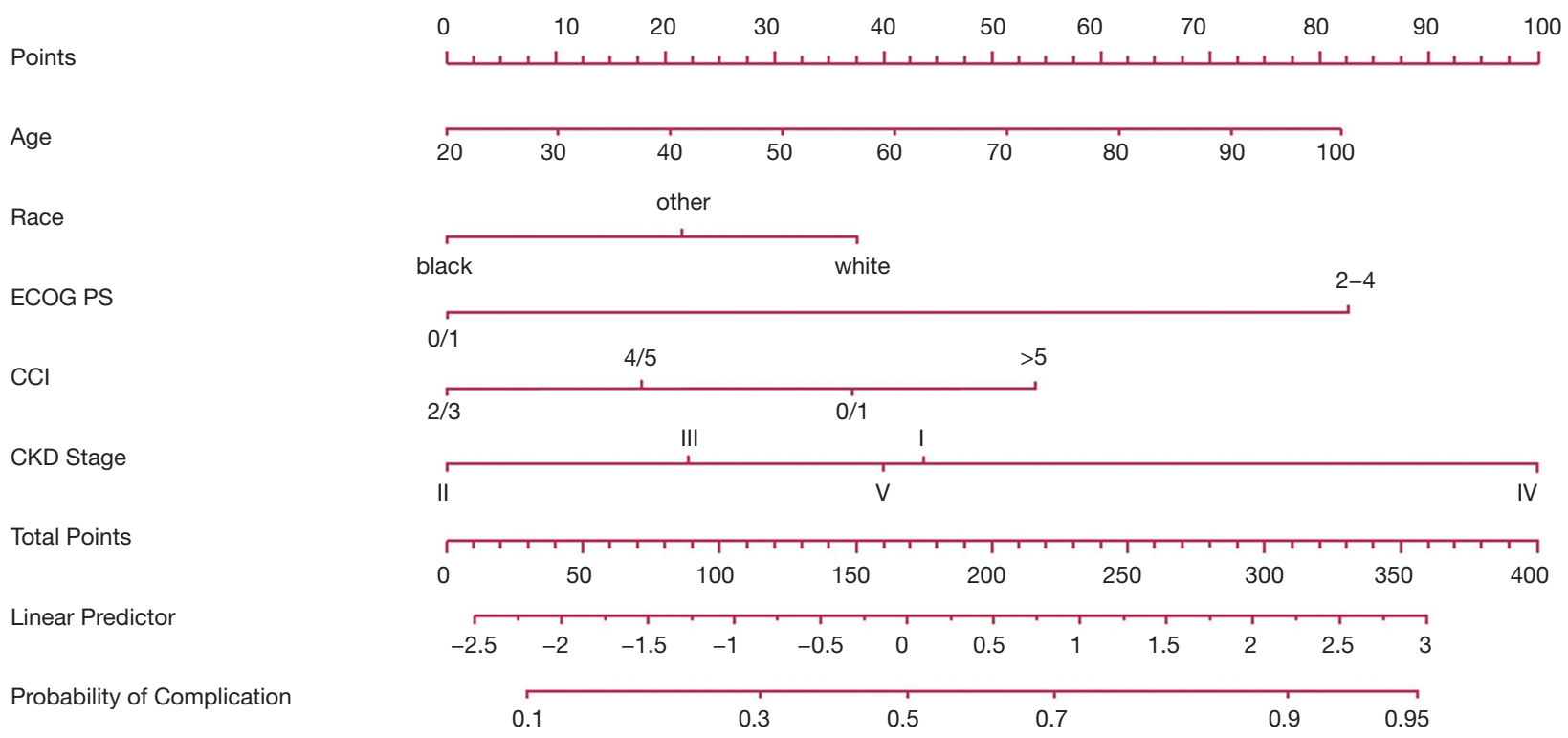

Figure 1 Nomogram predicting risk of perioperative complications within 30 days of radical nephroureterectomy.

cancer-specific and overall survival with a 5 -year overall survival of $47.5 \%$ in the low PNI group compared to $71.9 \%$ in the high PNI group. These differences remained significant on multivariate analysis. Using the NSQIP database and surrogate markers of nutrition status including hypoalbuminemia, preoperative weight loss, and low BMI, Katz et al. investigated the association with mortality and complications (11). On multivariate analysis these authors found significant association with hypoalbuminemia and 30-day mortality (OR 4.31) as well as likelihood of experiencing a complication (OR 2.09). In the absence of prospective studies the impact of improving a patient's nutritional status on post-operative recovery or mortality risk remains unclear. However, nutritional optimization is a potential direction for future research that could significantly impact patient outcomes.

\section{Perioperative complications}

RNU is an extensive urologic procedure in that the anatomical extent is broad, necessitating surgery in multiple abdominal quadrants. Compared to radical nephrectomy and partial ureterectomy, this increases the spectrum of risks and operative time. Due to increased complexity, adoption of fully intracorporeal minimally invasive techniques, compared to other urologic surgeries like prostatectomy and radical nephrectomy, was delayed.

Laparoscopic RNU was first published by Clayman et al. in 1991 (12). Initial series were concerning showing a worse safety profile for minimally invasive approaches when compared to the open standard of care. However, as operative techniques and experience has improved, modern series have reversed this trend. Since the introduction of robotic RNU there has been rapid adoption of this technique $(13,14)$. Our understanding of complications and sequelae of surgery are generally sourced from nonrandomized studies comparing the various surgical techniques.

\section{Retrospective studies}

Sugihara et al. published a large, propensity matched series comparing open and laparoscopic RNU using a Japanese claims database (15). This study included 6,944 patients from 2010-2012 but did not include robot-assisted cases. Patients undergoing laparoscopic RNU had lower baseline comorbidities, higher BMI, earlier oncologic stage, and were more likely to be performed in higher volume academic hospitals. After one-to-one propensity score matching based on preoperative characteristics 5,804 patients remained. Results significantly favored the minimally invasive approach in terms of postoperative complications, length of stay, and transfusion rate and a lower mortality rate was noted. Operative time was significantly shorter in the open group. They reported postoperative complication rate of $9.4 \%$ vs. $12.6 \%$ in the laparoscopic vs. open groups. Most 
frequent complications were cardiac events, GU infection, and sepsis/DIC. In both groups length of stay was longer (11 vs. 12 days) and transfusion rates higher (12\% vs. 20\%) than seen in contemporary cohorts. The benefit of this data is that it captures approximately $45 \%$ of inpatient hospitalizations in Japan. However, it excludes robotic cases, was populated for the purpose of payments and therefore may not accurately report complications, and may not be generalizable to other healthcare systems.

Recent publications have begun to investigate complication rates using a robotic approach, which is becoming increasingly more common. A study by Lee et al. retrospectively compared open, laparoscopic, and robotic RNU outcomes of 422 patients at a single center in South Korea (16). There was an impressive significant shift in surgical approach over the course of the study with a decrease in open surgery from $49.7 \%$ of cases from 2004-2009 to $6.8 \%$ from 2015-2017, and an increase in robotic approach from $2.4 \%$ in the early period to $77.4 \%$ in the later period. There were no differences in preoperative characteristics though malignant features were more aggressive in patients undergoing open surgery. Follow up was significantly longer in open surgery as expected, since more of these surgeries happened earlier. Surgical time was significantly different comparing robotic, laparoscopic and open RNU (248.5 vs. 230.2 vs. $210.5 \mathrm{~min}$ ). There was significantly less blood loss, shorter hospital length of stay, and less analgesic requirement using a minimally invasive approach. Postoperative overall complication rate ranged from $13.7-14.9 \%$ and was not significantly different between groups when complication severity was compared. The authors did not publish type and frequency of specific complications. This study is helpful in incorporating patients undergoing robotic and laparoscopic surgery but is limited by having not specified the most common complications by approach.

A multi-institutional series was published in 2019 by Campi et al. comparing robotic RNU to SU (17). The study focused on surgical feasibility as well as perioperative and oncologic outcomes of the procedures. They reported on 81 patients from three tertiary care centers between 2015-2018 with median follow up of 22 months. Preoperative characteristics suggest significant baseline comorbidities including a median eGFR of $61,65 \%$ current or former smokers, $51 \%$ with hypertension and $24 \%$ with preoperative cardiovascular events. Median operative time was 195 minutes and EBL was $200 \mathrm{cc}$. Median length of hospitalization was 5 days. The authors report that two patients $(3 \%)$ had intraoperative bleeding. Their overall complication rate was high at $44 \%$, the majority of which were Clavien grade 1 . Among more serious events were bleeding requiring epigastric artery embolization, percutaneous drainage of a symptomatic lymphocele, reintervention for bowel perforation and reintervention for large retroperitoneal hematoma. Two patients had delayed complications requiring incisional hernia repair. Median decrease in eGFR was 15 . Though this study was not randomized or prospective, it is a recent cohort therefore reflecting modern operative technique. It further benefits from chart review which improves its accuracy and value in reporting individual complications.

A population-based study of surgical outcomes was performed using the Premier Hospital Database by Tinay et al. (14). This database captures approximately $20 \%$ of discharges in the United States from about 600 hospitals and allows for longitudinal patient analysis. Over 34,000 patients were included from 2004-2013 undergoing RNU for UTUC, half of which were performed open. The total number of RNU decreased over the study period, which may suggest a shift towards renal sparing management (e.g., endoscopic, SU). Use of minimally invasive techniques increased over time, ultimately dominated by robotic approach. Complications were identified using ICD-9 codes then assigned Clavien classifications. No significant difference in overall mortality or major complications was identified when comparing approaches, however minor complications were more likely in the robotic group. The overall complication rate was lowest in the laparoscopic group (35.4\%) and highest in the robotic group (41.5\%); mortality rate ranged from $1.5 \%$ in the robotic group to $2.3 \%$ in the open group. Consistent with other studies, length of stay was lower and operative time was higher for minimally invasive RNU. The authors then limited their analysis to highest volume hospitals and surgeons and did not find any difference in morbidity or mortality. A more pronounced decrease in length of stay was seen in the minimally invasive RNU group, suggesting that complications are more attributable to the disease and surgery rather than the surgeon. This study is the largest we identified but is limited in relying on a retrospective billing database.

\section{Randomized trial}

To our knowledge only one randomized trial compares surgical approach when performing RNU. Simone et al. stratified 80 patients with non-metastatic UTUC by clinical stage and age then randomized them to undergo either open or laparoscopic RNU between 2003-2006 (18). 
Baseline comorbidities were not disclosed. Operative time was similar between groups but blood loss was significantly higher and length of stay was significantly longer in the open group. The mean follow-up time was 41 (range, 30-66) months. The authors reported 4 deaths in the open group and 8 in the laparoscopic group as well as higher metastatic rate in the laparoscopic group including 1 port site metastasis; however, in this small study these differences were not statistically significant. In contrast to the vast majority of similar RNU publications, the authors report no postoperative complications despite their extended follow up time. This data must be viewed with caution as it predates widespread mainstream adoption of laparoscopic nephroureterectomy, preoperative patient characteristics were not reported, and the complication rate is substantially lower compared to comparable studies, however, it remains the only publication that lacks the bias inherent to retrospective studies.

\section{Meta-analyses}

A meta-analysis was performed in 2012 by $\mathrm{Ni}$ and colleagues to compare laparoscopic versus open $\mathrm{NU}$ ultimately including 21 studies representing 1,235 laparoscopic RNU and 3,093 open RNU (19). They found longer operative time for laparoscopic RNU (241 vs. 203 minutes), but shorter length of stay (5.9 vs. 8.7 days) and significantly lower estimated blood loss (273 vs. $476 \mathrm{~mL})$. Complications were similar, favoring a minimally invasive approach. Intraoperative complications were rare and slightly lower in the MIS patients (4.4\% vs. $5.1 \%)$, and postoperative complications were also generally minor and infrequent (minor $5.7 \%$ vs. $7.8 \%$, major $4.6 \%$ vs. $3.8 \%$ ). The authors did find lower perioperative mortality in the open approach but this was also not statistically significant $(1.6 \%$ vs. $0.7 \%)$. Overall this publication suggests that both laparoscopic and open RNU are safe procedures. Types and frequency of complications were not reported.

Given the advances in surgical technique and learning curve that likely impacted earlier studies of minimally invasive RNU, Liu and colleagues performed an updated metaanalysis comparing laparoscopic to open RNU including studies from the preceding 10 years (2007-2017) (20). Twenty-five studies were analyzed including only a single randomized controlled trial. Consistent with other studies, laparoscopic RNU was a significantly longer operation [weighted mean difference (WMD) 44.85 minutes] and was associated with significantly shorter hospital length of stay (WMD -2.46 days). There was significantly lower blood loss from laparoscopic RNU (WMD -137.83 cc) and by extension lower rate of transfusion (OR 0.43). Seven studies reported complication rates and no significant differences were identified in terms of minor, major, or overall complications. This publication did not report specific complications and did not incorporate studies of robotic RNU.

\section{Renal function}

Loss of renal function is a major concern for patients with UTUC. Owing to the risk factors of developing urothelial tumors and older age, patients with UTUC are likely to have underlying renal dysfunction or comorbidities that predispose them to future dysfunction (21). Chronic kidney disease (CKD) is associated with substantial morbidity, including cardiovascular events, and mortality. CKD also limits a patient's ability to receive nephrotoxic chemotherapy in the future. Balancing the risk of loss of renal function with oncologic progression is extremely challenging.

$\mathrm{SU}$ is an option that is increasingly used in select patients in whom renal protection is desired and whose disease is surgically resectable. It has been suggested that the oncologic outcomes of SU in appropriately selected patients may be equivalent to RNU without significantly compromising renal function. Studies comparing $\mathrm{SU}$ to RNU help us to understand what the impact of these surgeries is on renal function. Fang et al. published a metaanalysis that evaluated renal function comparing RNU to SU in 2016 (22). Only retrospective studies were available for review. Of the 11 studies identified, 4 reported renal functional outcomes. The majority of studies reported similar baseline renal function between the RNU and SU groups. As expected, a significant decrease in eGFR of $9.32 \mathrm{~mL} / \mathrm{min} / 1.73 \mathrm{~m}^{3}$ in RNU compared to $\mathrm{SU}$ was demonstrated. Though statistically significant, the clinical impact of this change could not be explored in this analysis.

Taking another approach to address the question of renal loss after RNU after UTUC, Singla et al. evaluated changes in renal function in patients undergoing RNU compared to radical nephrectomy (23). This study is interesting in that it specifically addressed both the comorbidities inherent in patients who develop UTUC as well as surgical factors that go beyond the loss of a renal unit. The study cohort was comprised of 435 patients from 1997-2013 at a single center who underwent radical nephrectomy $(n=317)$ or nephroureterectomy $(\mathrm{n}=118)$. Patients were excluded if they underwent a nephron-sparing approach (endoscopic, 
SU), had neoadjuvant or adjuvant chemotherapy, or had bilateral disease. On average, RNU patients were older, had higher Charlson comorbidity index, worse ECOG performance status and were more likely to be tobacco users. Non-cancer-specific mortality was significantly higher in the UTUC group (11.9\% vs. $5.2 \%)$. Patients with UTUC had a significantly lower median baseline eGFR (58.4 vs. $74.9 \mathrm{~mL} / \mathrm{min} / 1.73 \mathrm{~m}^{2}, \mathrm{P}=0.003$ ) but interestingly experienced less loss of renal function compared to patients undergoing nephrectomy at first and last follow up. In nephrectomy patients, loss of renal function was significantly associated with non-cancer-specific mortality. One possible explanation for this finding is that the ipsilateral kidney has already sustained a preoperative functional loss, making postoperative decrease in function after removing the affected kidney less pronounced. This theory is supported by a subgroup analysis showing preoperative hydronephrosis being associated with less decline in eGFR; however, without functional studies this is purely speculative.

The role of cisplatin chemotherapy in the adjuvant and neoadjuvant setting is not yet clear, but is only available to patients with adequate renal function, limiting application in this high-risk population. To help identify patients with potential cisplatin eligibility, Lee et al. sought to determine which patients would be most likely to recover renal function after undergoing RNU (24). This single center, longitudinal study followed 118 patients for mean time of 10.8 months. Patients were broken into two groups based on a preoperative eGFR cutoff of $60 \mathrm{~mL} / \mathrm{min} / 1.73 \mathrm{~m}^{2}$, typically used as a cutoff for cisplatin eligibility. Patients in the low GFR group were significantly older and more likely to have hypertension. Rates of hydronephrosis were similar between groups. Unexpectedly, there was a significantly higher rate of renal function recovery in the low eGFR group. On multivariate analysis, the only other characteristic associated with renal function recovery was presence of hydronephrosis. This study suggests a counterintuitive effect of RNU on ultimate recovery of renal function that favors patients with lower preoperative function, but is limited by small size and short follow-up time.

\section{Conclusions}

RNU remains the standard of care for surgical treatment of UTUC but is associated with marked morbidity and nonnegligible risk of mortality in a population with significant baseline comorbidities. Preoperative patient optimization must be largely extrapolated from other studies and no specific interventions that benefit post-operative outcomes have been identified to date. Several tools are available to help guide the patient and surgeon when weighing the risks and benefits of proceeding to surgery in a particular patient. Adverse effect profiles do appear to be impacted by surgical approach but neither the open nor minimally invasive approach is clearly superior. High quality studies have been limited by low disease incidence and lack of prospective data collection. A deeper understanding of variables impacting complication rates for this rare disease entity would be greatly facilitated by a coordinated, multi-institutional, cooperative investigation in the future.

\section{Acknowledgments}

Funding: None.

\section{Footnote}

Provenance and Peer Review: This article was commissioned by the Guest Editors (John J. Knoedler and Jay D. Raman) for the series "Upper-Tract Urothelial Carcinoma: Current State and Future Directions" published in Translational Andrology and Urology. The article was sent for external peer review organized by the Guest Editors and the editorial office.

Conflicts of Interest: Both authors have completed the ICMJE uniform disclosure form (available at http:// dx.doi.org/10.21037/tau.2019.12.25). The series "UpperTract Urothelial Carcinoma: Current State and Future Directions" was commissioned by the editorial office without any funding or sponsorship. DC reports other from Cota Healthcare, outside the submitted work. The authors have no other conflicts of interest to declare.

Ethical Statement: The authors are accountable for all aspects of the work in ensuring that questions related to the accuracy or integrity of any part of the work are appropriately investigated and resolved.

Open Access Statement: This is an Open Access article distributed in accordance with the Creative Commons Attribution-NonCommercial-NoDerivs 4.0 International License (CC BY-NC-ND 4.0), which permits the noncommercial replication and distribution of the article with the strict proviso that no changes or edits are made and the original work is properly cited (including links to both the formal publication through the relevant DOI and the license). See: https://creativecommons.org/licenses/by-nc-nd/4.0/. 


\section{References}

1. Soria F, Shariat SF, Lerner SP, et al. Epidemiology, diagnosis, preoperative evaluation and prognostic assessment of upper-tract urothelial carcinoma (UTUC). World J Urol 2017;35:379-87.

2. Wein AJ, Kavoussi LR, Partin AW, et al. Campbell-Walsh Urology, Eleventh Edition. Philadelphia: Elsevier; 2016.

3. Flaig T, Spiess P. NCCN Clinical Practice Guidelines in Oncology: Bladder Cancer. 2019; Version 4.2019.

4. EAU Guidelines. Edn. presented at the EAU Annual Congress Copenhagen 2018. ISBN 978-94-92671-01-1.

5. Soodana-Prakash N, Balise R, Nahar B, et al. Perioperative outcomes and complication predictors associated with open and minimally invasive nephroureterectomy. Can J Urol 2018;25:9395-400.

6. Lascano D, Pak JS, Kates M, et al. Validation of a frailty index in patients undergoing curative surgery for urologic malignancy and comparison with other risk stratification tools. Urol Oncol 2015;33:426.e1-12.

7. Raman JD, Lin YK, Shariat SF, et al. Preoperative nomogram to predict the likelihood of complications after radical nephroureterectomy. BJU Int 2017;119:268-75.

8. Cui HW, Turney BW, Griffiths J. The Preoperative Assessment and Optimization of Patients Undergoing Major Urological Surgery. Curr Urol Rep 2017;18:54.

9. Richards T, Clevenger B, Keidan J, et al. PREVENTT: preoperative intravenous iron to treat anaemia in major surgery: study protocol for a randomised controlled trial. Trials 2015;16:254.

10. Huang J, Yuan Y, Wang Y, et al. Preoperative prognostic nutritional index is a significant predictor of survival in patients with localized upper tract urothelial carcinoma after radical nephroureterectomy. Urol Oncol 2017;35:671. e1-671.e9.

11. Katz M, Wollin DA, Donin NM, et al. Effect of Malnutrition on Radical Nephroureterectomy Morbidity and Mortality: Opportunity for Preoperative Optimization. Clin Genitourin Cancer 2018;16:e807-15.

12. Clayman RV, Kavoussi LR, Figenshau RS, et al. Laparoscopic nephroureterectomy: initial clinical case report. J Laparoendosc Surg 1991;1:343-9.

13. Rodriguez JF, Packiam VT, Boysen WR, et al. Utilization and Outcomes of Nephroureterectomy for Upper Tract Urothelial Carcinoma by Surgical Approach. J Endourol 2017;31:661-5.

14. Tinay I, Gelpi-Hammerschmidt F, Leow JJ, et al. Trends in utilisation, perioperative outcomes, and costs of nephroureterectomies in the management of upper tract urothelial carcinoma: a 10-year population-based analysis.
BJU Int 2016;117:954-60.

15. Sugihara T, Yasunaga H, Yu C, et al. Perioperative Outcome Comparisons Between Open and Laparoscopic Nephroureterectomy Among a Population-Based Cohort from 2010 to 2012. J Endourol 2015;29:770-6.

16. Lee H, Kim HJ, Lee SE, et al. Comparison of oncological and perioperative outcomes of open, laparoscopic, and robotic nephroureterectomy approaches in patients with non-metastatic upper-tract urothelial carcinoma. PLoS One 2019;14:e0210401.

17. Campi R, Cotte J, Sessa F, et al. Robotic radical nephroureterectomy and segmental ureterectomy for upper tract urothelial carcinoma: a multi-institutional experience. World J Urol 2019;37:2303-11.

18. Simone G, Papalia R, Guaglianone S, et al. Laparoscopic versus open nephroureterectomy: perioperative and oncologic outcomes from a randomised prospective study. Eur Urol 2009;56:520-6.

19. Ni S, Tao W, Chen Q, et al. Laparoscopic versus open nephroureterectomy for the treatment of upper urinary tract urothelial carcinoma: a systematic review and cumulative analysis of comparative studies. Eur Urol 2012;61:1142-53.

20. Liu F, Guo W, Zhou X, et al. Laparoscopic versus open nephroureterectomy for upper urinary tract urothelial carcinoma: A systematic review and meta-analysis. Medicine (Baltimore) 2018;97:e11954.

21. Hasan MN, Roupret M, Keeley F, et al. Consultation on UTUC, Stockholm 2018 aspects of risk stratification: long-term results and follow-up. World J Urol 2019;37:2289-96.

22. Fang D, Seisen T, Yang K, et al. A systematic review and meta-analysis of oncological and renal function outcomes obtained after segmental ureterectomy versus radical nephroureterectomy for upper tract urothelial carcinoma. Eur J Surg Oncol 2016;42:1625-35.

23. Singla N, Hutchinson R, Menegaz C, et al. Comparing Changes in Renal Function After Radical Surgery for Upper Tract Urothelial Carcinoma and Renal Cell Carcinoma. Urology 2016;96:44-53.

24. Lee BH, Zabor EC, Tennenbaum D, et al. Renal function recovery after radical nephroureterectomy for upper tract urothelial carcinoma. World J Urol 2018;36:257-63.

Cite this article as: Levy A, Canes D. Perioperative complications and adverse sequelae of radical nephroureterectomy. Transl Androl Urol 2020;9(4):1853-1859. doi: 10.21037/tau.2019.12.25 